\title{
Cytochrome $c$ oxidase assembly factors with a thioredoxin fold are conserved among prokaryotes and eukaryotes
}

\begin{abstract}
Cytochrome $c$ oxidase $(\mathrm{COX})$ is a multi-subunit terminal oxidase of the eukaryotic respiratory chain involved in the reduction of oxygen to water. Numerous lines of evidence suggest that the assembly of COX is a multi-step, assisted process that depends on several assembly factors with largely unknown functions. Sco1/2 proteins have been isolated as highcopy number suppressors of a deletion of copper chaperone Cox17, implicating Sco1/2 in copper transport to COX subunits I or II. Here I report the similarity of Sco1/2 assembly factors to peroxiredoxins and thiol:disulfide oxidoreductases with a thioredoxin fold, suggesting that Sco-related proteins perform a catalytic rather than a copper
\end{abstract}

\section{Y.V. Chinenov (}

Howard Hughes Medical Institute, University of Michigan Medical Center, 4570 MSRB II,

1150 W. Medical Center Drive,

Ann Arbor, MI 48109-0650, USA

e-mail: yurii@umich.edu

Tel.: +1-734-6152576

Fax: +1-734-6153397

Please send articles to:

Peer Bork

Max-Dehlbrück-Center

for Molecular Medicine (MDC)

Robert-Rössle-Strasse 10

D-13122 Berlin, Germany

and:

EMBL

Meyerhofstrasse 1

D-69117 Heidelberg, Germany

E-mail: bork@embl-heidelberg.de http://www.embl-heidelberg.de/ bork/ transport function. Reported sequence similarities, together with the functional role of bacterial Sco-related proteins suggest that Sco-related proteins represent a new class of membraneanchored thiol:disulfide oxidoreductases involved in COX maturation.

Key words $\mathrm{Sco} \cdot$ Cytochrome $c$ oxidase $\cdot$ Peroxiredoxins . Thioredoxin fold $\cdot$ Cox 17

\section{Abbreviations $C O X$ : Cytochrome $c$ oxidase}

Cytochrome $c$ oxidase (COX) is the terminal oxidase of the respiratory chain catalyzing the reduction of molecular oxygen to water with concomitant oxidation of cytochrome $c$. COX is a multi-subunit complex localized in the inner membrane of mitochondria or aerobic bacteria. Subunits I-III are conserved between prokaryotes and eukaryotes, and at least two of them (I and II) are required for catalytic activity [1]. Additional eukaryotic subunits may be involved in the modulation of activity, assembly, or tissue specific regulation of catalytic functions. Despite the considerable progress in elucidating the structural organization of COX [1] little is known about mechanisms of assembly of this large enzymatic complex. The presence of free subunits and partially assembled complexes suggests that COX assembly is a slow, sequential, and possibly assisted process [2, 3, 4]. More than 30 complementation groups deficient in $\mathrm{COX}$ activity have been identified in yeast. Most of these do not associate with known COX enzymatic activities, sug-
Bioinformatics: Bits and Bytes 8

gesting that assembly of mitochondrial complexes is dependent on a number of accessory proteins [5, 6]. Assembly factors may be responsible for the transport and insertion of heme (Cox10p, Cox11p), copper (Cox17p), or magnesium (Cox11p) into inactive enzymes, intermembrane subunit transport, and folding of COX subunits [7, 8, 9, 10, 11, 12].

Sco1p and the related Sco2p were originally identified as proteins required for COX assembly. Also, they are capable of partial suppressing COX17 deletion at elevated concentrations of copper $[7,8,9,10,11]$.

Cox $17 \mathrm{p}$ is an $8-\mathrm{kDa}$ protein containing a highly labile binuclear $\mathrm{Cu}$ (I)-thiolate cluster, presumably involved in copper delivery to COX II subunit $[4,7]$. Deletion of $\mathrm{SCO} 1$, but not $\mathrm{SCO} 2$, in yeast results in rapid degradation of both catalytic subunits of COX. The presence of CXXXC motif, similar to the CoxII copper binding site, led to the hypothesis that Scolp is also directly involved in copper transport $[10,12]$. Substitution of either of these cysteine residues results in the loss of active COX in yeast [10], demonstrating the importance of these residues. However, the requirement for elevated concentrations of copper suggests possible alternative functions of Sco1 and Sco2 in the assembly of COX.

PSI-BLAST [13] searches $(\mathrm{E}<0.001)$ against a nonredundant protein database revealed a significant similarity of Sco1p and Sco2p to several peroxiredoxines with known threedimensional structures and bacterial 


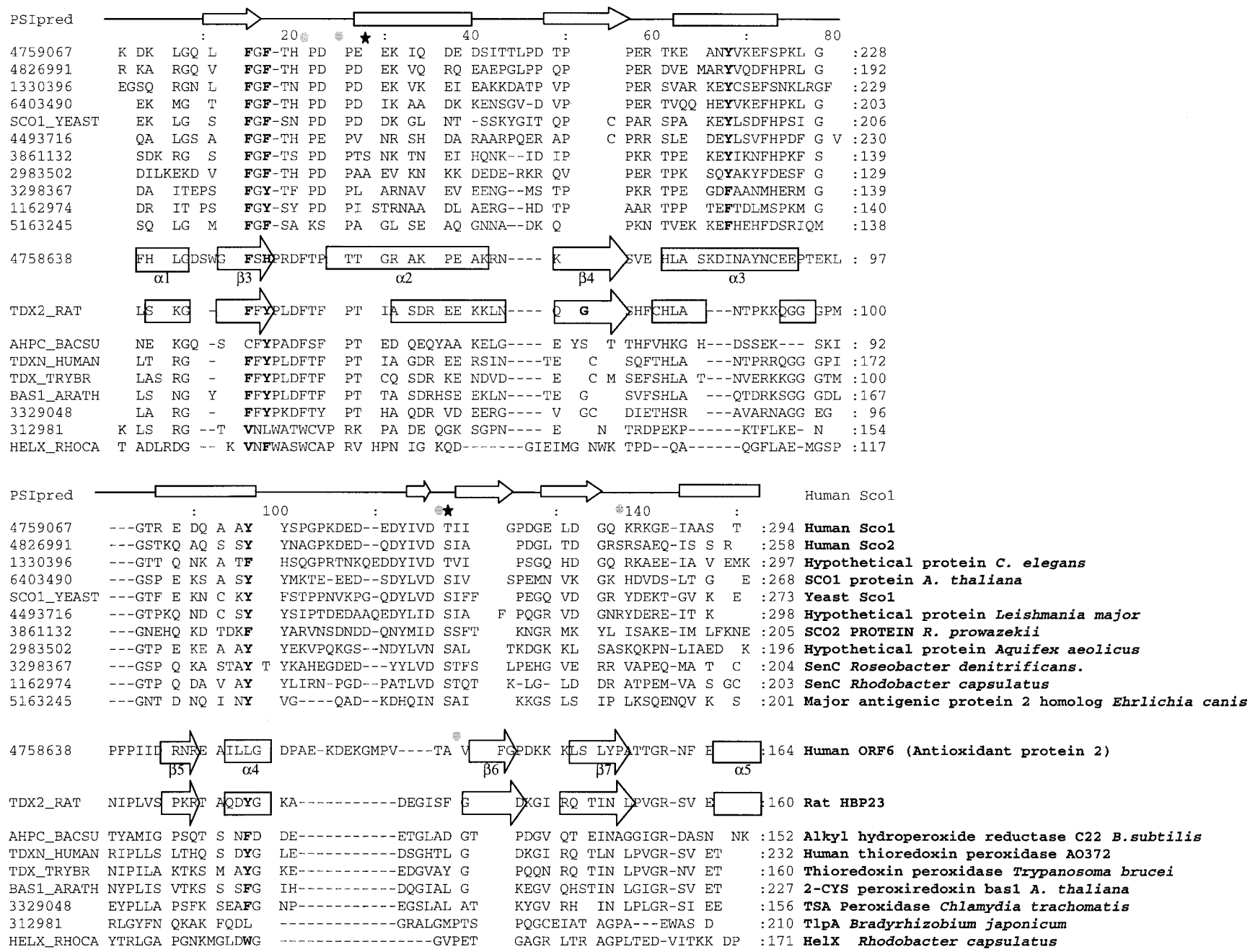

Fig. 1 Multiple alignment of Sco1-related proteins. A PSI BLAST search (E-value=0.001) of NCBI nonredundant protein database with the C-terminal portion of Saccharomyces cerevisae Sco1p revealed similarity to peroxiredoxins after the second iteration and bacterial thiol:disulfide oxidoreductases after the third iteration. Representative members of these groups were aligned using MULTALIN [14] with the following settings: symbol comparison table - BLOSUM 62, gap penalty 8, gap penalty at extension 0.05 . Aligned sequences were prepared for publication using GenDoc [31]. Conserved amino acids were colored according to the following scheme: dark blue hydrophobic residues

(ACFGHIKLMVWY); light blue aliphatic residues (ILV); gray aromatic residues (FHWY); red positively charged residues (KRH); purple DENQ; green polar (CDEHKNQRST); yellow small (ACDGNPSTV). Left column SwissProt protein names or GenBank identifier codes; right column amino acid positions are indicated for each protein; on the top of the alignment amino acid positions of aligned proteins; above the alignment with open rectangles secondary structure predicted with PSIpred [16], $\alpha$-helixes; open arrows $\beta$-strands. Secondary structure of human AOP2 (ORF6) and rat HBP23 is assigned from respective crystal structures and is shown as following: black rectangles $\alpha$-helices; black arrows $\beta$-strands. The annotation of secondary structure elements is as in crystal structure of ORF6 $[17,18]$. Orange circles residues of the putative active center and Arg-119 of the ORF6 active center; black stars above the alignment amino acid residues in the proposed Sco active center that are mutated in patients with infantile cardioencephalomyopathy [23]

thiol:disulfide oxidoreductases (Fig. 1). Although overall similarity is low, several regions in Sco1-related proteins and peroxiredoxin/thiol:disulfide oxidoreductase are conserved, including cysteine residues and hydrophobic residues in the active center (Fig. 1, residues 10-30). Representative proteins were aligned with MULTALIN [14]. The secondary structure predictions of yeast Sco1p using PHD and PSIpred algorithms $[15,16]$ are very similar to that of peroxiredoxins (hORF6 and rat HBP23 in Fig. 1) determined by X-ray crystallography $[17,18]$. The conserved regions include $\beta$-strand 3 , $\alpha$-helix 2, and a loop between them encompassing active center, $\beta$-strand 4 , and hydrophobic residues of $\beta$-strand 6 .

Peroxiredoxins and thiol:disulfide oxidoreductases reduce a wide range of substrates, including hydrogen peroxide, various organic peroxides, and disulfide bonds in proteins and low molecular weight compounds. The mechanism of substrate reduction involves an initial ionization of a cysteine residue in the active center (Fig. 1, Cys-25), followed by a nucleophilic attack of a substrate and formation of either mixed disulfide bonds or sulfenic acid [17, $19,20]$. The reduction of a mixed disulfide bond intermediate involves an attack by the second cysteine residue in the active center leading to the release 
of a reduced substrate and formation of intramolecular (thiol:disulfide oxidoreductase) or intermolecular (2-Cys peroxiredoxins) disulfide bond. Regeneration of the catalytically active reduced form of an enzyme probably involves a thioredoxin/thyoredoxin reductase system. In peroxiredoxins conserved Arg-119 (Fig. 1) abstracts a proton from the catalytic sulfhydryl group [17]. In hORF6 His-17 stabilizes the ionized sulfhydryl group. This position is occupied by an aromatic residue in most analyzed peroxiredoxins and Scorelated proteins, suggesting an alternative mechanism of thiolate intermediate stabilization [18]. In HBP23 interactions between Arg-119, Glu-28, and Asp-137 coordinating a putative $\mathrm{Cl}$ anion in a close proximity to catalytic cysteine are implied in the activation of Cys- 25 and stabilization of a reactive thiolate intermediate [18]. Although a position equivalent to Arg-119 is not conserved among Sco-related proteins, a conserved histidine residue located nearby (Fig. 1, His-117) may be involved in the activation of a sulfhydryl group in a predicted active center. Activation of the catalytic sufhydryl group by a histidine has been previously described for papain and Yersinia PTPase [21, 22]. Glu-28 and Asp-137 are conserved in almost all analyzed Scorelated proteins. Recent findings have implicated the substitution of Glu-28 to lysine in human Sco2 as a cause of fatal infantile cardioencephalomyopathy [23]. A second patient with mutated Sco2 in this study had Ser-118 substituted by phenylalanine. Similarly to yeast with deleted Sco1, both patients displayed marked reduction in COX activity in heart and muscles and reduction in content of mtDNA-encoded COXI and COXII subunits [23]. Thus mutations at or near residues in the predicted Sco active center detrimentally affect Sco2 function and cytochrome $c$ oxidase assembly in human.

Several bacterial thiol:disulfide oxydoreductase, including Rhodobacter capsulatus helX, Bradyrhizobium japonicumu cycX, and Bradyrhizobium japonicum TlpA (Fig. 1), have been implicated in cytochrome $c$ and COX biogenesis, presumably maintaining cysteine residues of the apocytochrome $c$ heme-attachment site in reduced state
$[24,25,26]$. Since cytochrome $c$ biogenesis is not affected in $\mathrm{scol}$ or $\mathrm{sco} 2$ mutants, the primary target of Sco must be elsewhere. The ability of Sco1 to suppress cox 17 deficiency of copper transport suggests a possible function downstream in the COX assembly pathway, probably on the step concomitant or immediately preceding copper insertion. Physical interactions of Cox $17 p$ and Sco1/2p were recently demonstrated in a high throughoutput two-hybrid screening for interacting yeast proteins [27]. Although spontaneous incorporation of copper into CoxII $\mathrm{CuA}$ center is possible, it requires high concentrations of copper and rather nonphysiological $\mathrm{pH}[28,29,30]$. The formation of Cox $17 \mathrm{p} / \mathrm{Sco} 1 / 2 \mathrm{p}$ could facilitate a coordinated reduction of cysteine residues in the CoxII metal-binding center with copper insertion. Therefore, when the delivery of copper to copper-binding centers of Cox II is impaired due to Cox $17 \mathrm{p}$ deficiency, overexpressed Sco1p increases the probability of either spontaneous copper incorporation or utilization of alternative copper transporters. The ubiquity of Sco-related proteins and suggests that the mechanism of COX assembly is well-conserved between kingdoms.

Acknowledgements I am grateful to Mensur Dlakić, Asya Grinberg, Mark E. Martin, and Chris Coombs for critical reading of the manuscript and for insightful discussion.

\section{References}

1. Michel H, Behr J, Harrenga A, Kannt A (1998) Cytochrome c oxidase: structure and spectroscopy. Annu Rev Biophys Biomol Struct 27:329-356

2. Wielburski A, Nelson BD (1983) Evidence for the sequential assembly of cytochrome oxidase subunits in rat liver mitochondria. Biochem J 212:829-834

3. Nijtmans LG, Taanman JW, Muijsers AO, Speijer D, Van den Bogert C (1998) Assembly of cytochrome-c oxidase in cultured human cells. Eur J Biochem 254:389-394

4. Grivell LA, Artal-Sanz M, Hakkaart G, de Jong L, Nijtmans LG, van Oosterum K, Siep M, van der Spek H (1999) Mitochondrial assembly in yeast. FEBS Lett 452:57-60
5. McEwen JE, Ko C, KloecknerGruissem B, Poyton RO (1986) Nuclear functions required for cytochrome c oxidase biogenesis in Saccharomyces cerevisiae. Characterization of mutants in 34 complementation groups. J Biol Chem 261:11872-11879

6. Tzagoloff A, Dieckmann CL (1990) PET genes of Saccharomyces cerevisiae. Microbiol Rev 54:211-225

7. Srinivasan C, Posewitz MC, George GN, Winge DR (1998) Characterization of the copper chaperone Cox17 of Saccharomyces cerevisiae. Biochemistry 37:7572-7577

8. Schulze M, Rodel G (1989) Accumulation of the cytochrome c oxidase subunits I and II in yeast requires a mitochondrial membrane-associated protein, encoded by the nuclear SCO1 gene. Mol Gen Genet 216:37-43

9. Glerum DM, Shtanko A, Tzagoloff A (1996) SCO1 and SCO2 act as high copy suppressors of a mitochondrial copper recruitment defect in Saccharomyces cerevisiae. J Biol Chem 271:20531-20535

10. Rentzsch A, Krummeck-Weiss G, Hofer A, Bartuschka A, Ostermann K, Rodel G (1999) Mitochondrial copper metabolism in yeast: mutational analysis of Scolp involved in the biogenesis of cytochrome c oxidase. Curr Genet 35:103-108

11. Paret C, Ostermann K, Krause-Buchholz U, Rentzsch A, Rodel G (1999) Human members of the SCO1 gene family: complementation analysis in yeast and intracellular localization. FEBS Lett 447:65-70

12. Buggy J, Bauer CE (1995) Cloning and characterization of senC, a gene involved in both aerobic respiration and photosynthesis gene expression in Rhodobacter capsulatus. J Bacteriol 177:6958-6965

13. Altschul SF, Madden TL, Schaffer AA, Zhang J, Zhang Z, Miller W, Lipman DJ (1997) Gapped BLAST and PSIBLAST: a new generation of protein database search programs. Nucleic Acids Res 25:3389-3402

14. Corpet F (1988) Multiple sequence alignment with hierarchical clustering. Nucleic Acids Res 16:10881-10890

15. Rost B, Sander C (1993) Prediction of protein secondary structure at better than 70\% accuracy. J Mol Biol 232:584-599

16. Jones DT (1999) Protein secondary structure prediction based on positionspecific scoring matrices. J Mol Biol 292:195-202

17. Choi HJ, Kang SW, Yang CH, Rhee SG, Ryu SE (1998) Crystallization and preliminary X-ray studies of hORF6, a novel human antioxidant enzyme. Acta Crystallogr D Biol Crystallogr 54:436-437 
18. Hirotsu S, Abe Y, Okada K, Nagahara $\mathrm{N}$, Hori H, Nishino T, Hakoshima T (1999) Crystal structure of a multifunctional 2-Cys peroxiredoxin heme-binding protein $23 \mathrm{kDa} /$ proliferation-associated gene product. Proc Natl Acad Sci USA 96:12333-12338

19. Chivers PT, Raines RT (1997) General acid/base catalysis in the active site of Escherichia coli thioredoxin. Biochemistry $36: 15810-15816$

20. Holmgren A (1985) Thioredoxin. Annu Rev Biochem 54:237-271

21. Lewis SD, Johnson FA, Shafer JA (1981) Effect of cysteine-25 on the ionization of histidine-159 in papain as determined by proton nuclear magnetic resonance spectroscopy. Evidence for a his-159-Cys-25 ion pair and its possible role in catalysis. Biochemistry 20:48-51

22. Zhang ZY, Dixon JE (1993) Active site labeling of the Yersinia protein tyrosine phosphatase: the determination of the $\mathrm{pKa}$ of the active site cysteine and the function of the conserved histidine 402 . Biochemistry 32:9340-9345
23. Papadopoulou LC, Sue CM, Davidson MM, Tanji K, Nishino I, Sadlock JE, Krishna S, Walker W, Selby J, Glerum DM, Coster RV, Lyon G, Scalais E, Lebel R, Kaplan P, Shanske S, De Vivo DC, Bonilla E, Hirano M, DiMauro S, Schon EA (1999) Fatal infantile cardioencephalomyopathy with COX deficiency and mutations in SCO2, a COX assembly gene. Nat Genet 23:333-337

24. Beckman DL, Kranz RG (1993) Cytochromes $\mathrm{c}$ biogenesis in a photosynthetic bacterium requires a periplasmic thioredoxin-like protein. Proc Natl Acad Sci USA 90:2179-2183

25. Ramseier TM, Winteler HV, Hennecke H (1991) Discovery and sequence analysis of bacterial genes involved in the biogenesis of c-type cytochromes. J Biol Chem 266:7793-7803

26. Loferer H, Bott M, Hennecke H (1993) Bradyrhizobium japonicum TlpA, a novel membrane-anchored thioredoxinlike protein involved in the biogenesis of cytochrome aa3 and development of symbiosis. EMBO J 12:3373-3383

27. Uetz P, Giot L, Cagney G, Mansfield TA, Judson RS, Knight JR, Lockshon D, Narayan V, Srinivasan M, Pochart P, Qureshi-Emili A, Li Y, Godwin B, Conover D, Kalbfleisch T, Vijayadamodar G, Yang M, Johnston M, Fields S, Rothberg JM (2000) A comprehensive analysis of protein-protein interactions in Saccharomyces cerevisiae Nature 403:623-627
28. Lappalainen P, Aasa R, Malmstrom BG, Saraste M (1993) Soluble CuAbinding domain from the Paracoccus cytochrome c oxidase. J Biol Chem 268:26416-26421

29. Slutter CE, Sanders D, Wittung P, Malmstrom BG, Aasa R, Richards JH, Gray HB, Fee JA (1996) Water-soluble, recombinant $\mathrm{CuA}$-domain of the cytochrome ba3 subunit II from Thermus thermophilus. Biochemistry 35:3387-3395

30. Hay M, Richards JH, Lu Y (1996) Construction and characterization of an azurin analog for the purple copper site in cytochrome c oxidase. Proc Natl Acad Sci USA 93:461-464

31. Nicholas KB, Nicholas HB Jr, Deerfield DWI (1997) GeneDoc: Analysis and Visualisation of Genetic Variation. http://www.cris.com/ $\sim$ Ketchup/genedoc.shtml 